\title{
A muscle-like recruitment actuator with modular redundant actuation units for soft robotics
}

\author{
Glenn Mathijssen ${ }^{\mathrm{a}, \mathrm{d},}$, Joshua Schultz ${ }^{\mathrm{b}, \mathrm{c}}$, \\ Bram Vanderborght ${ }^{\mathrm{a}}$, Antonio Bicchi ${ }^{\mathrm{b}, \mathrm{d}}$ \\ ${ }^{a}$ Department of Mechanical Engineering, Vrije Universiteit Brussel (VUB), B-1050 \\ Brussels, Belgium \\ ${ }^{b}$ Department of Advanced Robotics, Istituto Italiano di Tecnologia (IIT), 16163 Genova, \\ GE, Italy \\ ${ }^{c}$ Department of Mechanical Engineering, the University of Tulsa, Tulsa, OK 74104, USA \\ ${ }^{d}$ Bioengineering and Robotics Research Center, Centro E. Piaggio, Universita di Pisa, \\ 56112 Pisa, PI, Italy
}

\begin{abstract}
Human muscles contrast sharply with traditional robot actuators in that they consist of several motor units, connected in series and parallel, which can be progressively recruited. Some roboticists have explored this idea in robotic actuators, striving for improvements such as the ability to withstand partial damage, inexpensive repeatability by discrete open loop control, the potential of modular actuators, etc. These systems, however, become rather complex or rely on less widely used actuation techniques such as piezo-actuators or SMAs to produce a compact implementation. This paper presents a novel design of a modular redundant actuation unit which can be combined in various combinations to form compliant actuators with varying characteristics. The actuation unit consists of discretely activated solenoids with an integrated compliant coupling. This paper presents the working principle and the physical implementation in detail. Failure of a single motor unit will merely lead to a loss in performance rather than failure of the actuator. Since each motor unit is discrete, neither power electronics nor control require analog signals. Isometric experiments display the actuation characteristics and demonstrate the repeatability. The platform can
\end{abstract}

\footnotetext{
* Corresponding author

Email address: Glenn.Mathijssen@vub.ac.be (Glenn Mathijssen)
} 
be used in future work to further explore the virtues of exploiting discretization and redundancy in muscle-like control.

Keywords: Discrete actuation, Modular motor units, Soft robotics, Redundant actuation

2014 MSC: 00-01, 99-00

\section{INTRODUCTION}

Reproducing the properties of biological muscle is a longstanding research effort since actuator limitations heavily influence the capabilities of robots. Stiffness properties of muscles is one of the earliest characteristics that has been

5 studied. The seminal work of Pratt et al. from the 1990s [1] showed the virtue of elastic elements in series with the drive train and ever since numerous roboticists have focused on novel uses and implementations of series elastic actuators (SEA). An important extension of this work towards muscle-like actuators is the work on variable impedance actuators (VIA) [2, 3]. Vanderborght et al. [4]

10 provides a good review with classification of actuators that can vary stiffness and damping characteristics so as to exploit and modify the natural dynamics of a system. In addition, dedicated control architectures for safety [5] and energy efficiency [6] are developed. A number of materials and techniques have been explored for their use in artificial muscles, such as pneumatic artificial muscles 15 7], electroactive polymers (EAP) 8] [9], shape memory alloys (SMA) [10], etc.

Apart from its compliance, the hierarchical structure of skeletal muscle is also distinctive, though this aspect has received less attention. A skeletal muscle consists of multiple motor units, each consisting of a number of muscle fibers. Each motor unit can be activated through its motor neuron: as such a muscle 20 with more motor units is able to control force output in a finer manner. The activation of motor units to produce a force is called motor unit recruitment and differs markedly from generating an analog signal proportional to the desired force output [11] 12]. Actuators with a discrete cellular structure include the work of Dittrich [13], MacNair and Ueda [14] and Huston et al. [15]. Both 
25 of these works present a type of actuator that is made up of numerous subactuators. This redundancy increases the robustness of these actuators. Failure of an electromechanical component will only lead to a loss in performance instead of a loss of a complete degree of freedom (DOF), since the remaining undamaged units can continue to perform the task. The cellular structure opens the

30 possibility for modularity in the actuators which represents an untried frontier in engineering. In Mathijssen et al. [16] the series-parallel elastic actuation concept was proposed whereby multiple springs in parallel can be variably recruited by multiple dephased intermittent mechanisms and only one motor. Cho et al. 17] introduced and validated a segmented cellular architecture of SMA wires.

35 Ueda et. al [18] focused on distributed stochastic control of an actuator system consisting of many cellular SMA units. Schultz and Ueda [19] validated their multilayer strain amplification mechanism on a camera positioning mechanism based on piezo actuators. For mathematical simplicity, each active element of the actuator in Schultz and Ueda's actuator was identical and interchangeable.

40 Most mechatronic devices composed of modular units are in the context of self-organizing systems [20] [21], distributed manipulation [22] or modular actuators for multi-DOF systems [23]. Using modular components to precisely configure the actuation system for a particular performance characteristic for a given joint axis has been less studied. Most actuators are size selected rather

45 than built-to-order. This work presents a modular actuation unit which can be combined in series and parallel combinations. Each actuation unit contains 12 motor units which can be discretely activated similarly to motor units in the human body. Since this actuation unit is based on activation of individual motor units, it generates contractions under the motor unit recruitment paradigm.

50 This paper begins to explore the idea that the central nervous system deliberately chooses activation patterns with motor units of different characteristics [24]. The actuation unit pictured in Fig.1 has a compliant coupling mechanism specifically constructed so as to produce different operating characteristics from physically identical active elements, which was first introduced in [25]. We ${ }_{55}$ present here a new spring design with an improved manufacturing process, bet- 


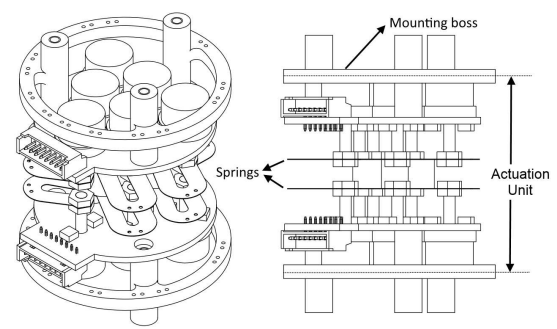

Figure 1: Single degree of freedom actuation unit consisting of discretely activated elements coupled to the mounting boss by a spring. This actuation unit is a building block for constructing designer muscle-like actuators with a hierarchical structure. Such actuators are controlled by setting an activation level, or the number of active elements in the on state, a process known as recruitment.

ter repeatability and greater ease of assembly. An experimental validation of the spring models is reported in the paper, showing a good match between our mathematical models and physical device behaviors. Furthermore, we present the results of isometric experiments on actuators that consist of different arrangements of actuation units. Finally, we have quantified variability over the various choices of activation patterns for two representative configurations.

Section 2 discusses the most important concepts and key terms with regard to this paradigm. The actuation unit implementation is discussed in Section 3 , More specifically the implementation of solenoids, springs and straps (mechan65 ical stops), close packing of motor units, and custom drive circuits is discussed. The results of different actuator configurations composed of the actuation units are presented in Section 4 . Section 5 concludes the paper and potential future work is proposed.

\section{KEY TERMS AND WORKING PRINCIPLE}

Beyond those described in Section1, the authors are not aware of applications where modules are combined based on a performance characteristic for a particular joint axis. Section 2 recaps the key terms for modular actuation introduced in [25], discusses the importance of compliance, and introduces the functional model of the actuation unit shown in Fig. 1. 
The following terms are introduced or borrowed (with some license) from biology, and their specific meanings in the context of this paper delineated:

- Cellular [26] muscle-like actuator: a motion or force producing device composed of more than one actuation unit and containing more than one motor unit.

- Actuation unit: this is a manufacturing distinction. It refers to the smallest possible unit that can be conveniently added, removed or reconfigured to adjust the muscle-like actuator's characteristics.

- Motor unit [11]: this is computational or communication distinction. It refers to the collection of force-producing devices that can be independently activated or deactivated by a single communication line.

Actuation units' and motor units' physical boundaries may coincide, but are not required to. Several paradigms are delineated in [25] and shown in Fig.2] A schematic of the paradigm implemented in this paper is shown in Fig.2a 90 and Fig.2b, Each actuation unit consists of multiple motor units, which can be activated independently. The implementation, to be described in Section3, has each motor unit corresponding to one solenoid in series with a compliant element. Each actuation unit has multiple solenoids in parallel.

\subsection{Compliance enables discretization}

Skeletal muscle fibers are arranged in parallel in sufficient numbers to generate the required force. A single muscle fiber does not necessarily run the entire length of the muscle, so series connections are also important [27]. The same reasoning is valid for modular actuators where actuation units are connected in series and parallel. The compliance between these units is indispensable to the operation of a modular actuator. This can be understood by a thought experiment: take two units tied together in parallel with no compliant interface but instead a rigid end cap. The contraction of one unit would then result in the 


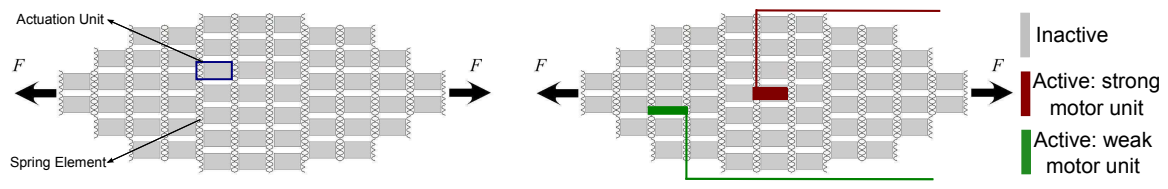

(a) A muscle-like actuator consisting (b) One actuation unit is formed by several motor of modular actuation units. units, each controlled by a signal line.

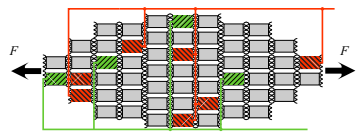

(c) One motor unit is formed by some collection of actuation units, and can be activated by a signal line.

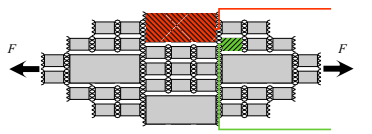

(d) One motor unit is formed by one actuation unit.

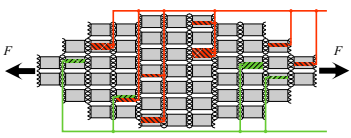

(e) Motor units are dispersed among several actuation units.

Figure 2: Different paradigms for actuation units and motor units, adapted from [25]. (a) shows a muscle-like actuator composed of discretely activated actuation units coupled by compliant elements. (b) - (e) show different paradigms, with in each example one strong and one weak motor unit active. The paradigm implemented in this work is shown in (b), where each actuation unit consists of multiple motor units. The signal lines to inactive motor units have been omitted for clarity. 
same contraction of the other unit. Any other case would violate the compatibility condition of mechanics of materials [28]. This is indicated in Fig. 3. By connecting a contractile unit (analogous to a muscle fiber) through a compliant connection, rather than a rigid one, the compatibility condition is satisfied. Instead of requiring displacements to be equal, the compliant material imposes a mathematical relationship between displacement and the force contribution of that fiber. In skeletal muscle, the fibers are instead connected together by endomysial connective tissue [27], which is elastic. In modular actuation units, this elastic force-displacement relationship can be exploited and specified so as to obtain desirable properties for the muscle-like actuator as a whole.
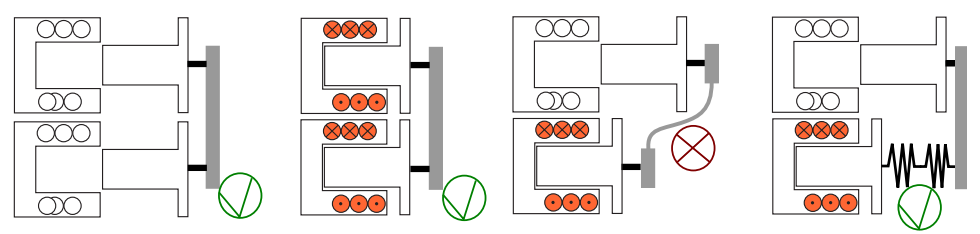

Figure 3: The compliant element is crucial to allow different contraction patterns amongst actuation units. The three most left configurations do not include a compliant element. The third figure indicates the inconsistency, which is resolved by the compliant element in the fourth figure.

\subsection{Working principle of an actuation unit}

The working principle of an actuation unit of the type described in this work 115 is explained by means of the functional model in Fig.4 This is an example of the paradigm in Fig.2 The implementation is further explained in Section3.

Each functional part of the actuation unit schematic is indicated in Fig.4a For clarity, the schematic consists of only 2 parallel solenoids which function as the motor units; in practice, any number may be present. Since a solenoid is a unidirectional actuator, the actuation unit is unidirectional as well. Both solenoids are fixed to a printed circuit board (PCB) and connected to a leaf spring. Since the displacement is the same, the stiffness of the spring distinguishes between strong and weak motor units. The stiffness of each spring is 
named $k_{w}$ and $k_{s}$ respectively. The stroke $L_{x}$ of the solenoid is indicated as well.

${ }_{125}$ When the plunger is pulled away from the coil, a strap becomes taut and prevents the plunger from separating from the coil. Each strap is fixed to the PCB and to the middle of one of the springs. The ends of the springs opposite of the plunger are fixed to the mounting boss. When a solenoid contracts it deforms the spring, which results in a force at the mounting boss. Each combination of a solenoid and a spring can, as such, be considered an SEA. Adjacent actuation units connect at the mounting boss and ultimately, to the robotic link itself. The working principle of an actuation unit will be explained via Fig. $4 \mathrm{~b}, 4 \mathrm{c}$,

- If an actuation unit experiences an extension $(x<0)$ as in Fig. $4 \mathrm{~b}$ and Fig. $4 \mathrm{c}$ both active and inactive motor units contribute to the force at the mounting boss. An inactive motor unit will behave as spring due to the taut strap. Each spring whose strap is engaged will have a stiffness equal to a fraction $f$ of the stiffness of the spring, i.e. $f k_{w}$ or $f k_{s}(0<f<1)$, depending on the connection of the strap to the springs, which determines the number of springs that can extend. The connection of the strap differs from that in 25]. An active motor unit will contribute with a stiffness equal to the full stiffness of the spring, i.e. $k_{w}$ or $k_{s}$. In Fig.4b both units are inactive and behave like a spring with stiffness $f k_{w}$ and $f k_{s}$, since part of the spring is being cut off and unstrained. In Fig.4c both the inactive and active unit contribute to the force at the output $F_{a}$.

- If an actuation unit experiences a contraction $(x>0)$, the inactive motor units will float and generate no output force $F_{a}$. The active motor units will contribute a stiffness equal to the full stiffness of the spring, i.e. $k_{w}$ or $k_{s}$

The schematic in Fig. 4 shows only 2 solenoids, each having a different spring 150 stiffness $k_{w}$ and $k_{s}$. In a more general case, each actuation unit consists of $N$ motor units, of which $\frac{N}{2}$ motor units have a spring stiffness $k_{s}$ and $\frac{N}{2}$ motor units have a spring stiffness $k_{w}$. All solenoids are identical; functionally they act as a displacement source. The characteristics of the spring element a given 


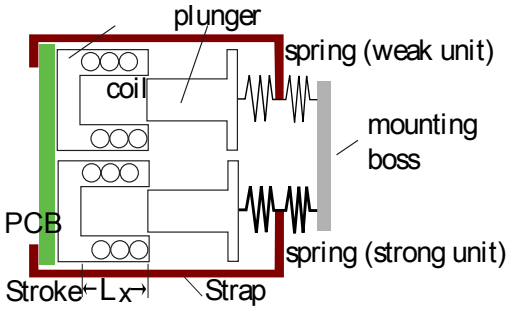

(a) Actuation unit schematic.

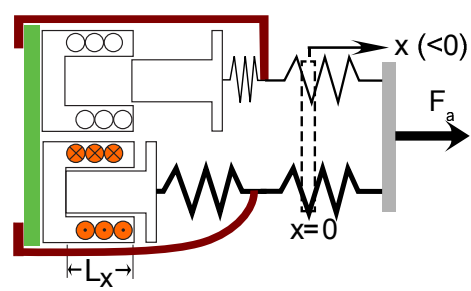

(c) Active: extension $(x<0)$.

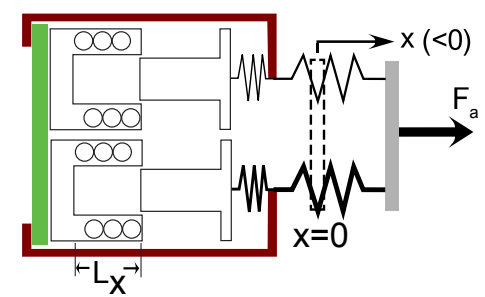

(b) Inactive: spring-like behavior $(x<0)$.

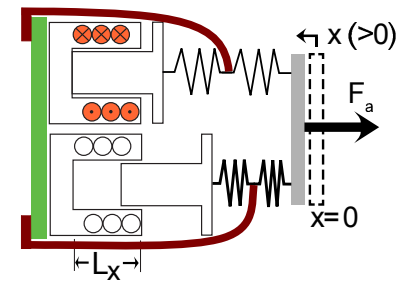

(d) Active: contraction $(x>0)$.

Figure 4: Actuation unit concept schematic; only a single strong and a single weak motor unit are shown for clarity. The important conceptual parts are indicated in (a) which shows an inactive unit under zero load. The springs are at their resting length. In both (b) and (c) the displacement $x$ is negative, subsequently referred to as an extension. As a result, both the active and the inactive motor units contribute to the output force $F_{a}$. The strap of the strong motor unit in (c) becomes limp due to the active motor unit and the extension. The contraction in (d) results in 2 limp straps and as a result only the active motor units contribute to the output force $F_{a}$. 
solenoid is connected to determines whether it is a strong or weak motor unit.

155 The spring path to the mounting boss is stiffer for the strong motor unit than for the weak, resulting in a higher force for the same solenoid displacement. It is easy to generalize this concept to actuators which posses finite numbers of actuation levels greater than 2 ; there would simply be more grades of stiffness. Ideally, the geometry is chosen such that

$$
k_{\text {weak }}=r k_{\text {strong }}, r \in \mathbb{R} / \mathbb{Q}, 0<r<1
$$

$$
F=\left\{\begin{array}{l}
\left(L_{x}-x\right)\left(M_{w} k_{w}+M_{s} k_{s}\right), x \geq 0 \\
\left(L_{x}-x\right)\left(M_{w} k_{w}+M_{s} k_{s}\right)-x\left(\left(\frac{N}{2}-M_{w}\right) f k_{w}+\left(\frac{N}{2}-M_{s}\right) f k_{s}\right), x<0
\end{array}\right.
$$

It should be noted that selecting a specific activation pattern also results in a change in stiffness of the actuator. Depending on which motor units (strong 
or weak) and how many are activated, and which straps and how many are taut, the stiffness of the actuator will change. Rigorous study of this property is beyond the scope of this article and will be addressed in a future work.

\section{ACTUATION UNIT IMPLEMENTATION}

In this section the physical implementation of the actuation units is discussed. The general schematic of an actuation unit, discussed in the previous

185 Section 2.3 , consists of one PCB and $N$ solenoids with $\frac{N}{2}$ strong and $\frac{N}{2}$ weak motor units. As defined in Section 2.1, the term actuation unit is a manufacturing distinction. In this work, two PCBs with 6 solenoids each (3 weak and 3 strong motor units) are combined to form an actuation unit. A spring element is placed in series with each plunger, and connected to the mounting boss. The

190 spring stiffness of the weak and strong motor units is respectively defined as $k_{w}$ and $k_{s}$. The following main parts of an actuation unit will be discussed in the subsections hereafter: solenoids which should be arranged optimally, springs and straps, and the custom drive circuit.

\subsection{Solenoids}

195 The contractile element of the motor unit is a miniature solenoid; each actuation unit contains twelve. This solenoid is produced by the Line Electric Company, S. Glastonbury, CT, USA, and claims to be "the world's smallest solenoid". The form factor of this solenoid is the same as a TO-5 transistor package and is particularly suited to the construction of compact actuation units. Performance specifications of the solenoid are listed in Table1, Each solenoid can be independently activated, making it a convenient analog to a biological motor unit. Active materials such as electroactive polymers [9] and shape memory alloy (SMA) [10] could also be used.

The solenoids consist of two parts, the coil and the plunger. If sufficient current is present in the coil, the plunger will be drawn into the coil until it reaches the ferrous rear end of the coil. If there is insufficient current present, 
Table 1: TO-5 solenoid performance specifications. Peak force assumes the plunger is at $80 \%$ of fully contracted.

\begin{tabular}{c|c|c} 
Quantity & Value & Unit \\
\hline Stroke Length & 2.54 & $\mathrm{~mm}$ \\
Peak Force & 1.6 & $\mathrm{~N}$ \\
Steady State Force & 1 & $\mathrm{~N}$ \\
Response time (max) & 0.5 & $\mathrm{~ms}$ \\
Cross Section & 65.4 & $\mathrm{~mm}^{2}$ \\
Coil Resistance & 6 & $\Omega$ \\
Weight & 1.7 & $\mathrm{~g}$ \\
\hline
\end{tabular}

the plunger will float $(x>0)$ or rest against the strap $(x \leq 0)$ as shown in Fig.4. The coils are soldered to a custom PCB which manages electrical connections to each solenoid, while structurally coupling them together rigidly. The unoccupied space between the solenoids for the outer ring is used for the mounting holes. A tab is added on one side to accommodate a Molex Picoblade 8-pin connector. A rigid standoff mounted to the printed circuit board allows the connection of additional modules in series.

\subsection{Motor unit arrangement by close packing}

In order to approximate a continuous signal by recruiting solenoids, there must be a reasonably large number of motor units within the allotted space. For a given number of motor units, the best motor unit density is achieved by close-packing the solenoids, which have a circular cross section. To facilitate the design of the spring element, and to keep it symmetric, the solenoids were placed in two concentric rings, with the strong motor units in the outer ring, and the weak ones in the inner ring. This is advantageous from a power standpoint, because the stronger motor units will require more current. Placing them on the outside will better allow them to dissipate heat. This is not a biologically inspired arrangement; the situation in biology is much more complicated and is motivated by additional factors not represented in this design, such as routing 
of nerves and blood vessels. In fact, the individual muscle fibers from various motor units are interspersed with one another 29].

The smallest non-trivial example contains 6 motor units: 3 strong and 3 weak motor units. This arrangement is illustrated in Fig.5a. The centroids of the inner ring of solenoids lie on a circle of radius $r$, and the centroids of the outer ring of solenoids lie on a circle of radius $R$. The circumscribing circle of the outermost ring, of radius $C$, represents the minimum theoretical radius of any actuator of this type. They can be calculated from the number of solenoids as follows:

$$
\begin{array}{r}
r=\frac{d}{2 \sin \frac{\pi}{n}} \\
R=\frac{d \sqrt{3}}{2}+r \cos \frac{\pi}{n} \\
C=R+\frac{d}{2}
\end{array}
$$

where $d$ denotes the diameter of the solenoid, and $n$ denotes the number of solenoids in the inner ring. Thus the motor unit density per unit area for this configuration, $\sigma$, can be calculated as:

$$
\sigma=\frac{n}{\pi C^{2}} .
$$

This can be used as a metric to evaluate the relative merits of a given muscle-like solution and helps the designer answer the following question: Is the actuator getting larger in order to increase the control resolution or the actuator maximum force? It should be noted that Eq. (6) is only a theoretical value, and due to manufacturing tolerances and other design considerations, the actual value of the finished device may in fact be lower.

It turns out that this is not the most compact regular close-packed arrangement; a single ring of 6 is actually more compact [30]. A single ring of 6 solenoids would pose difficulty with regard to the spring design, however. The relative compactness of arranging the motor units in a single circle or two concentric 


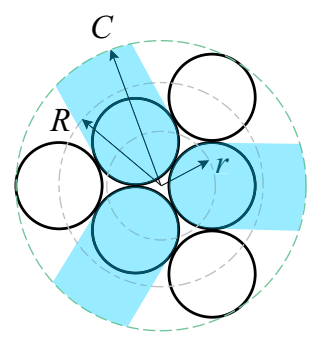

(a) Close-packed solenoid arrangement, two motor unit levels. Shaded region indicates area allotted to the spring element for the weak motor units.

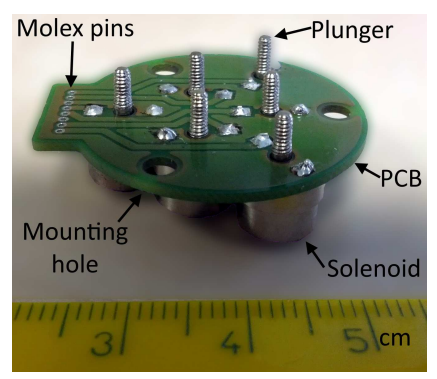

(b) Picture of the PCB with 6 solenoids mounted on and the plungers installed.

Figure 5: Schematic (a) and picture (b) of the solenoid arrangement that was implemented in this work.

circles can be determined by comparing the quantity $r_{2 n} / R_{n}$,

$$
\frac{r_{2 n}}{R_{n}}=\sqrt{3} \sin \frac{\pi}{2 n}+\cos \frac{\pi}{2 n}-\frac{1}{2 \cos \frac{\pi}{2 n}}
$$

where the subscript $n$ denotes the number of motor units in the innermost ring. Note that this index does not depend on the motor unit diameter, d. Eq. (7) converges to 2 as $n$ approaches $\infty$. By means of Eq. (7) it can be shown that two concentric rings become a more compact solution once the device contains 10 units [30]. When the number of concentric rings is greater than 2 , and the number of motor units becomes larger, the situation becomes more complicated, as closer packings are possible [31] with the concentric rings having different numbers of motor units. In fact, it has been shown that in many cases asymmetric packings are the most dense [32], 33]. This would, however, make it very difficult to design a suitable spring element, and would cause undesirable off-axis bending and possible alignment issues in manufacturing and operation.

The space within the envelope (be it a circle of radius $C$ or otherwise) circumscribing the motor units but not occupied by the solenoids themselves is not necessarily wasted space. In some cases, this space could be used for drive electronics, sensing, or computational elements, making the device more of an integrated solution. 
The spring element is not simply a series elastic element, but rather is crucial to the operation of the device, as it allows some solenoids to be active and others inactive at the same time. As alluded to in Section2.2, the spring element serves several functions: it combines the various efforts of the motor units at the output, presents a compliant interface with the environment, and since the solenoids correspond to a displacement source, determines which solenoids correspond to strong motor units and which correspond to weak motor units.

The selection of this spring element represents a significant engineering challenge, because it must accomplish the following objectives. It must:

- Not significantly decrease the strain rate $\epsilon$ of the actuator.

- Not interfere mechanically with other motor units when transitioned between the active and inactive states.

- Be able to be manufactured without using exotic or costly manufacturing techniques.

- Fit within (or at least not greatly exceed the dimensions of) the circumscribing circle of radius $C$, described in Section 3.2 .

Each of these objectives becomes more difficult to accomplish with decreasing size. To maximize the strain rate, the spring element should be as thin as possible in the actuation direction. The following Sections 3.3.1 and 3.3.2 report on two custom made designs based on different manufacturing techniques: a wire form spring and a leaf spring. The wire form spring was presented in [25], but details were omitted due to the short format, they are presented here.. In Section 3.3.1 the connection issues of the lobes to form the wire form spring are discussed as well. In Section 3.3.2 the leaf spring, an improved spring design, is introduced and the characterization experiments are reported. In Section 3.3 .3 the implementation of the straps is delineated. 


\subsubsection{Wire form spring}

The wire form spring consists of 3 weak motor unit springs and 3 strong motor unit springs, further referred to as weak and strong lobes. Although both lobes are custom wire forms, their design was chosen to make sure the spring would be manufacturable by conventional methods. The weak lobe is a basic oval-shaped coil, as shown in Fig.6a, The shape is as such that it fits in one of the three shaded areas in Fig. 5 a . The shaded area was arbitrarily allotted to the weak lobe. This is admittedly a heuristic, but logical based on the needs of the manufacturing process. The longitudinal axis of the oval is aligned with the radial direction of the circular PCB. The radius of the circular ends, $R_{w}$ will be the same as the radius of the solenoid, based on the region available. $L$ is chosen so that one circular end coincides with the solenoid itself, whereas the other is tangent to the outer envelope of the actuator. Then the number of coils, $n_{c}$ required to get the desired stiffness $k$ can be calculated using Eq. (8):

$$
k=\frac{d_{w}^{4} E \pi}{64 n_{c}\left(\frac{L^{3}}{3}+(1+\nu) R_{w}^{2}\left(L+\pi R_{w}\right)\right)}
$$

where $E$ is the elastic modulus, $d_{w}$ is the wire diameter and $\nu$ is the Poisson's ratio for the material. Eq. 8 is derived from Castigliano's theorem so that the deformation of the stroke of the solenoid plunger (for the strong coil) would result in a force equal to the pull force of the solenoid coil. Castigliano's theorem 310 is a standard method for the design of deformable structures; the interested reader may consult [34] for details and examples. Bending in the straight section of length $L$ and torsion in all sections were considered in the straight segment; the curved segment did not consider bending. Bending in the straight section accounts for $13.7 \%$ of total deflection, a small but not insignificant amount. If the number of coils is small, the spring element will be nearly planar, giving this design a clear advantage with regard to strain rate over designs using commercial springs.

The strong motor unit's spring must not encroach within the shaded area in Fig. 5a lie within the circle described in Section 3.2. contain the center of 
the solenoid to which it is attached within the interior of the coil, and have few enough coils so as to be considered thin, i.e., it must fit conveniently within the remaining available space. The natural shape to fit the space is an oval, but with the longitudinal axis perpendicular to the radial direction rather than aligned with it.

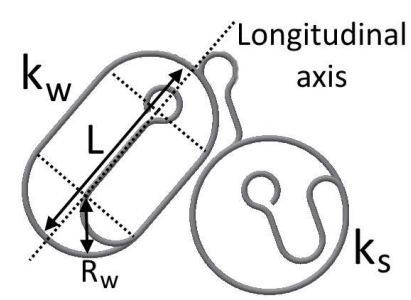

(a) 1 weak and 1 strong lobe. Manufactured in 1 piece.

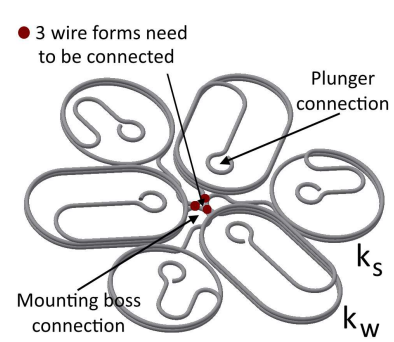

(b) 3 pairs of 1 weak and 1 strong lobe, connected together to form the spring.

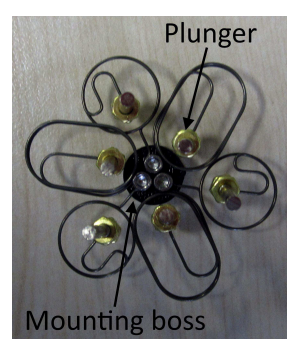

(c) Picture of the wire form spring.

Figure 6: Drawings and a picture of the wire form spring introduced in 25]. free parameters, as well as the oval's center, that can be used to select spring performance subject to the constraints. A numerical constrained optimization was conducted to determine the values that give the coil geometry with the minimum number of coils for the desired stiffness. The result, not surprisingly, the shaded region $\left(R_{w}=5.58 \mathrm{~mm}\right)$. The required $n_{c}$ is 1.6 .

The selected wire diameter is 25 AWG or $0.455 \mathrm{~mm}$. This resulted in a theoretical strong lobe stiffness of $0.1926 \frac{\mathrm{N}}{\mathrm{mm}}$ and a weak lobe stiffness of $0.1352 \frac{\mathrm{N}}{\mathrm{mm}}$. The deflection predicted by Eq. (8) agrees with that predicted by finite element methods within 19\%, indicating that the model described by Eq. (8) is useful for constructing a spring element that a spring element that will give close to the maximum holding force of the solenoid without overheating. The custom wire forms are provided by the Active Spring Company, Sibley's Green, United Kingdom.

One disadvantage is that the complete wire form spring (with 6 lobes) cannot 
be manufactured in a single piece, in a single operation. In this implementation 3 wire forms, each containing one strong lobe and one weak lobe need to be connected. This was done by M1 screws and a precisely manufactured clamp, and later by a micro welding process. Although feasible and validated empirically, both techniques are unreliable and significantly increase the complexity of the wire form spring. The clamp needs to be of high precision since the wire diameter is small and is fastened by M1 screws. The micro welding degrades the metallurgical qualities of the springs. Furthermore, the repeatability of the manufacturing process (including the connection) is rather low and clear differences could be observed. Finally, the adjustment cost to produce a variant of the wire form spring is relatively high. The aforementioned issues motivated us to search for modifications that would improve manufacturing consistency and efficiency of the process. Therefor, the next Section 3.3.2 discusses a new spring design that solves the encountered issues of the wire form spring.

355 3.3.2. Novel leaf spring

The general idea is to have a central hub, from which 6 beams emanate toward each solenoid. The spring, with 6 lobes, is laser-cut in a single piece out of a thin spring steel sheet. This process proved to be highly repeatable and precise. Furthermore, the laser cutting technique allows for easy connection to the straps, mounting boss and plungers as indicated in Fig.7b. The challenge is to make the beams compliant enough, since the actuator diameter is small and thus the beams cannot be long, increasing the bending stiffness. Furthermore, the space is limited so the lobes cannot bend back and forth many times in the plane. In order to retain the same compact solenoid arrangement, a dropin replacement for the wire spring was required, placing additional constraints on the design. Appropriate stiffness and extension can, however, be achieved by laser cutting U-shaped lobes out of a $0.1 \mathrm{~mm}$ thin 1.413 Chromium-nickel austenitic stainless spring steel plate (AISI 301). A schematic and picture are shown in Fig.7] The precise lasers of Raytech, Brugge, Belgium allowed for a 370 lobe width $w$ of $2.4 \mathrm{~mm}$. The density $\rho$ of AISI 301 steel is $7900 \mathrm{~kg} / \mathrm{m}^{3}$, elastic 


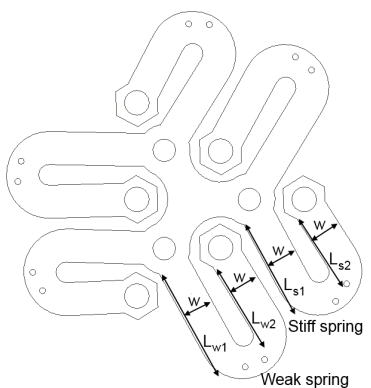

(a) Leaf spring schematic indicating the design parameters.

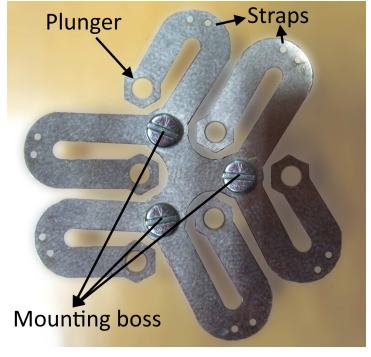

(b) Picture of the leaf spring indicating the connections to plungers and mounting boss.

Figure 7: Leaf spring design with 6 U-shaped lobes cut out of a $0.1 \mathrm{~mm}$ spring steel sheet.

modulus $E$ of $189 \mathrm{GPa}$, yield strength $\sigma_{y}$ of $1072 \mathrm{MPa}$ and tensile strength $\sigma_{t}$ of $1309 \mathrm{MPa}$. A schematic of the leaf spring design and illustration of the lengths of the weak and strong U-shaped lobes, $L_{w 1}, L_{w 2}, L_{s 1}$ and $L_{s 2}$, is shown in Fig.7a

The analytical model to calculate the force produced by a lobe $F_{\text {lobe }}$ as a function of the extension $\Delta x$, consists of standard beam bending of both of the legs of the spring as shown in Eq. (9). It is assumed that the vertical force $F_{\text {lobe }}$ applied at the plunger connection and the bend of the U-shape. Euler-Bernoulli bending in the straight sections is assumed, and deformations in the curved sections are neglected.

$$
F_{\text {lobe }}=\Delta x \frac{3 E I}{L_{1}{ }^{3}}+\Delta x \frac{3 E I}{L_{2}{ }^{3}}
$$

As noted in Table1, the maximum steady state force of the TO-5 solenoid is $1 \mathrm{~N}$ when fully contracted. Over a stroke length of $2.54 \mathrm{~mm}$ this results in a maximum linear spring stiffness of $0.39 \mathrm{~N} / \mathrm{mm}$. A safety factor of 3 is included to account for potential friction in the system. The turn of the U-shaped lobe is slightly larger than the PCB to allow for connection to the straps. Taking into account these goals and geometrical constraints, the parameters of the strong and weak lobe were tuned which resulted in the following specifications: 


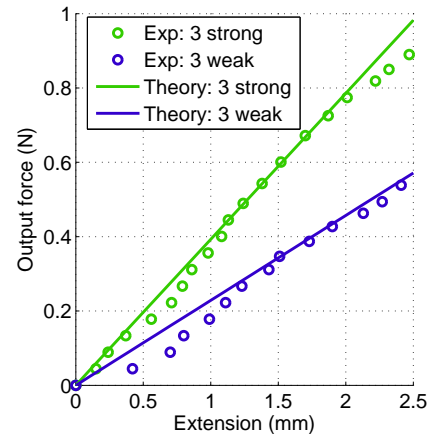

(a) Leaf spring $w=2.4 \mathrm{~mm}$.
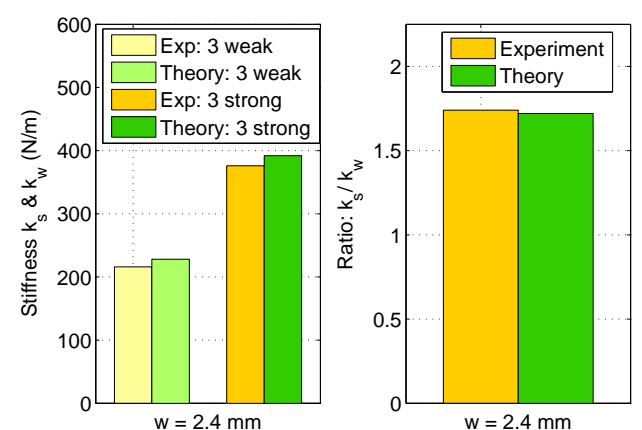

(b) Compare linear stiffness and stiffness ratio theoretically and experimentally.

Figure 8: Measurements characterizing and comparing stiffness of the leaf spring with $w=$ $2.4 \mathrm{~mm}$. The theoretical model and the experimental data match fairly well.

- Strong lobe: $w=2.4 \mathrm{~mm}, L_{s 1}=9.9 \mathrm{~mm}, L_{s 2}=7.5 \mathrm{~mm}$. Theoretical stiffness: $0.13 \mathrm{~N} / \mathrm{mm}$.

- Weak lobe: $w=2.4 \mathrm{~mm}, L_{w 1}=11.5 \mathrm{~mm}, L_{w 2}=9.1 \mathrm{~mm}$. Theoretical stiffness: $0.08 \mathrm{~N} / \mathrm{mm}$.

The spring stiffness of the weak and strong lobes were calculated theoretically and verified experimentally. The results are summarized in Fig.8a, The stiffness profile and stiffness ratio between the strong and weak lobe are compared in the bar charts of Fig.8b, From both figures it is clear that the theoretical model and the measurements match fairly well. These measurements are repeated for $w=2.8 \mathrm{~mm}$ and $w=3.2 \mathrm{~mm}$. In both cases the results were similar and consistent to those for $w=2.4 \mathrm{~mm}$, reported in Fig. 8

\subsubsection{Straps}

In Section 2.3 the function of the straps is introduced. The straps in the actuation unit presented in this work are made of 4 strand braid, including 3 dyneema $\AA$ strands, with a diameter of $0.25 \mathrm{~mm}$. As explained in Fig. 4 the straps should be loose when the mounting boss is compressed (with respect to the neutral position), and taut when the mounting boss is extended to prevent 


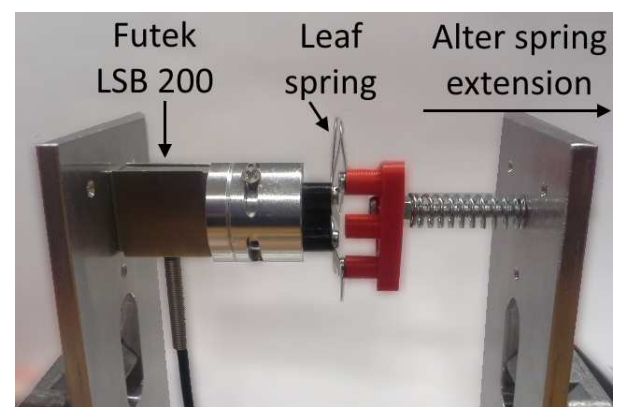

Figure 9: Leaf spring experimental to determine the stiffness profile of the leaf spring.

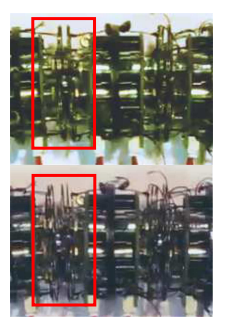

(a) Unactuated

(top) and actu-

ated (bottom)

unit.

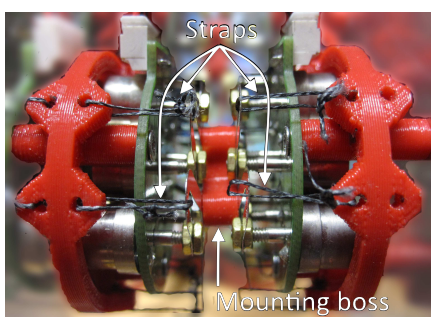

(b) Loose strap when mounting boss is compressed.

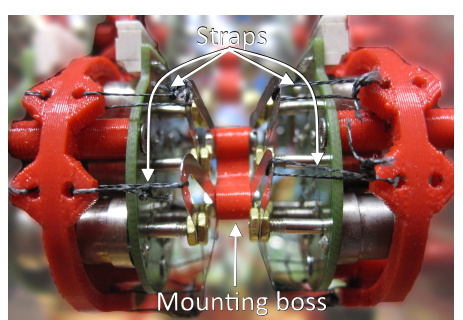

(c) Tensed strap when mounting boss is extended.

Figure 10: Working principle and implementation of the straps.

the plungers from leaving the coils. Both cases are illustrated respectively in

400 Fig.10b and Fig.10c. The assembly of the straps was performed by hand. As can be seen in Fig.10, the straps are connected to the standoffs (red), which are fixed to the PCB, and to the bend of the U-shaped lobes. Two actuated (bottom) and unactuated (top) units in order to visualize the spring extension, which is accentuated in red in Fig.10a,

\subsection{Custom drive circuits}

Custom drive circuits activate each of the 12 solenoids in the actuation unit independently. The activation is initiated by a digital signal. An activation consists of 2 phases. Initially, a high voltage (15 V) is applied across the solenoid for a short duration $(20 \mathrm{~ms})$. This results in a burst of current $(3 \mathrm{~A})$, which ensures 
that in the worst-case scenario (isometric contraction) the solenoid plunger will still reach the ferrous rear plate of the coil. This closes the magnetic flux gap, increasing the total force. After this short burst, the voltage drops $(2.5 \mathrm{~V})$ and a steady state current of approximately $0.5 \mathrm{~A}$ ensures the plunger is held in the solenoid coil while avoiding overheating of the coil.

The custom drive circuits are activated and deactivated by means of digital I/O lines of the National Instruments USB-6501 DAQ modules. One actuation unit requires 12 digital I/O lines to independently control the 12 solenoids. The DAQ modules can be controlled by a Labview interface or matlab code.

\subsection{Actuation unit performance specifications}

Performance specifications of a single actuation unit, as shown in the sketch in Fig.1 and in the picture in Fig.10b, are listed in Table2, Although the objective is not to exactly copy the human muscle, comparing to the performance specifications of mammalian muscle is still interesting. Based on [35] it can be concluded that the strain rate $\epsilon$ of the actuation unit $(21.1 \%)$ is comparable 425 to mammalian skeletal muscle $(20 \%)$. The maximum stress of the actuation unit $(2.3 \mathrm{kPa})$, however, is a factor 40 lower compared to mammalian skeletal muscle $(0.1 \mathrm{MPa})$. Performance specifications of actuators composed of these actuation units, can then be derived from the values in Table2 In general, the maximum force is multiplied by the number of strings in parallel, the maximum stroke length is multiplied by the number of actuation units in series, and $P_{s s}$ and the mass are multiplied by the number of actuation units. To put these performance specifications into perspective, they can be compared to the nested piezoelectric cellular actuators discussed in [19]. The strain rate of the actuation unit and the cellular actuators is comparable, and respectively equal to $21.1 \%$ and $20 \%$. The maximum stress the actuation unit can apply is $2285 \mathrm{~Pa}$, which is over 4 times the capability of the cellular actuator that can approximately apply $539 P a$. 
Table 2: Actuation unit with leaf spring of $w=2.4 \mathrm{~mm}$ : performance specifications

\begin{tabular}{c|c|c} 
Quantity & Value & Unit \\
\hline Maximum stroke length & 5 & $\mathrm{~mm}$ \\
Maximum force & 1.55 & $\mathrm{~N}$ \\
Response time (max) & 0.5 & $\mathrm{~ms}$ \\
Strain rate $\epsilon$ & 21.1 & $\%$ \\
$\sigma_{\text {block }}$ & 2285 & $\mathrm{~Pa}$ \\
Steady State Power $P_{s s}$ & 12.5 & $\mathrm{~W}$ \\
Mass & 37 & $\mathrm{~g}$ \\
\hline
\end{tabular}

\section{EXPERIMENTAL VALIDATION OF ACTUATION UNITS}

The aim of this Section 4 is to study the isometric output force of the actuation units in several representative configurations. More specifically, a chain of 4 units in series (further referred to as $1 \times 4$ actuator) will be compared to a bundle of 2 parallel chains of 2 actuation units (further referred to as $2 \times 2$ actuator). A study on the repeatability of the output force as a function of the activation patterns is presented as well.

445 4.1. Experimental set-up and activation graph

The $1 \times 4$ and $2 \times 2$ actuator assemblies are shown in Fig.11a and Fig.11b respectively. Both actuators were isometrically fixed between two rigid supports. A Futek LSB200 force sensor (maximum load $1 \mathrm{lb}$ ) was placed between the actuator and one of the rigid supports to measure the output force generated ${ }_{450}$ by the actuator. A Futek CSG110 signal conditioner/amplifier was used along with the LSB200 force sensor to generate an analog output signal. The data acquisition was performed by a National Instruments USB-6000 DAQ module and custom Labview code.

Since the straps between each spring and the mounting feature, were set to ${ }_{455}$ be $2 \mathrm{~mm}$ when taut, the equilibrium length of the $1 \times 4$ actuator is $16 \mathrm{~mm}$ and $8 \mathrm{~mm}$ for the $2 \times 2$ actuator. Both actuators were isometrically fixed with $25 \%$ 


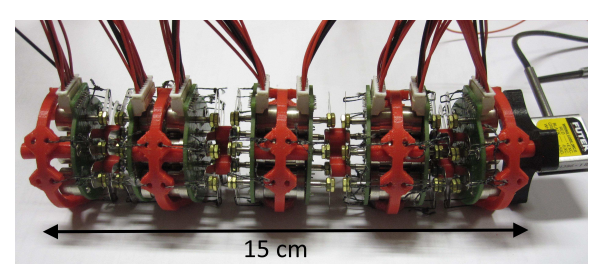

(a) $1 \times 4$ actuator: a chain of 4 actuation units in series.

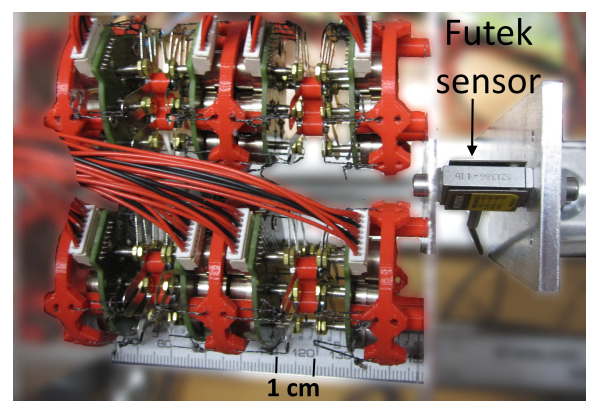

(b) $2 \times 2$ actuator: a bundle of 2 parallel chains of 2 actuation units.

Figure 11: Pictures of the $1 \times 4$ and $2 \times 2$ actuators.

pretension ( $4 \mathrm{~mm}$ and $2 \mathrm{~mm}$, respectively). This pretension serves to return the plunger to its initial position after contraction, overcoming friction. Moreover, the pretension ensures all straps are taut to cancel strap length variations due to the manufacturing process by hand. The pretension force was measured to be approximately $0.1 \mathrm{~N}$ and $0.2 \mathrm{~N}$, respectively for the $1 \mathrm{x} 4$ and $2 \times 2$ actuator. In the experimental results the pretension force is subtracted from the results. In future applications this pretension is to be expected since these actuation units are designed to be used in an antagonistic configuration.

Both the $1 \times 4$ and $2 \times 2$ actuators consist of 4 actuation units, for a total of 48 solenoids. Each solenoid can be independently activated by means of 2 National Instruments NI USB-6501 DAQ modules, along with the custom drive electronics. An example of an isometric output force graph over time is shown in Fig.12. The activation of each individual solenoid at $100 \mathrm{~ms}$ intervals is clearly visible. A longer steady-state output force is maintained (for 600ms) after an additional solenoid is activated on each PCB. The data was filtered with a second-order Butterworth filter with a normalized cut-off frequency of 0.05. It is important here to note that activating each solenoid in succession is just one option. Multiple, or even every single solenoid can be activated at once. This makes for higher peak currents, however. As a result, the actuator can in principle reach any isometric output force almost instantaneously, similar 


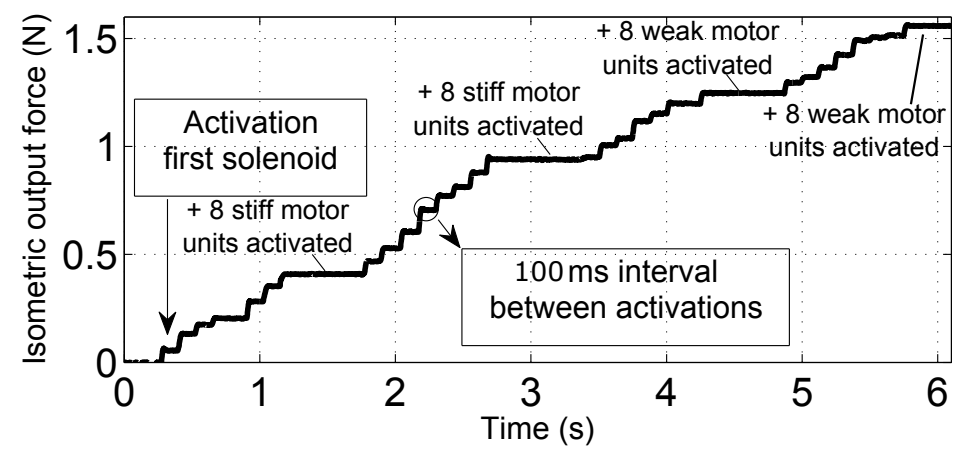

Figure 12: An isometric output force graph over time where the activation of each individual solenoid is clearly illustrated.

electric motors, which have a small electrical time constant.

\subsection{Isometric output force experiments}

The isometric output force experiments consist of activating a certain number of motor units on each of the 8 PCBs. This is performed for both the $1 \times 4$ and $2 \times 2$ actuator. The results are shown by means of 2 bar charts in Fig.13. With a total of 48 motor units, it is impractical to present all conceivable activation patterns. The first 2 bars in Fig.13a for example present the activation of 1 weak motor unit on each PCB. Since each of the 8 PCBs has 3 weak motor units, there are $3^{8}$ or 6561 different, though redundant, activation patterns possible. By means of a random generator, 10 different activation patterns were fired and each bar in Fig.13 shows the mean isometric output force of 10 different activation patterns. The constant force due to the pretension is subtracted from the results in order to compare the results between different experiments. The standard deviation is shown in red on top of each bar.

The bars in Fig.13a show the results of the activation of 1 weak motor unit on each PCB, 2 weak motor units, 1 strong motor unit, and 2 strong motor units. It is clear that the ratio between the activation of 1 weak (strong) motor unit and the activation of 2 weak (strong) motor units on each PCB is approximately $495 \quad 0.5$, which is to be expected since the force in parallel is doubled. The same goes for the ratio between the activation of a certain number of solenoids on each 


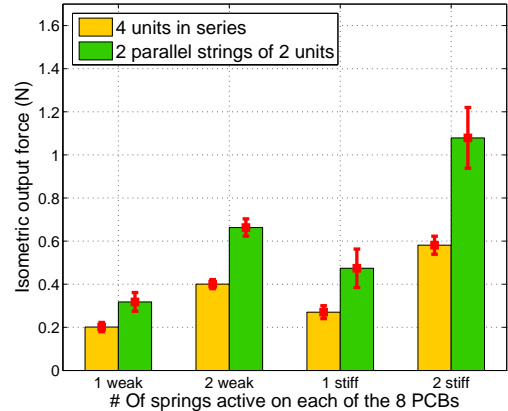

(a) Activating a certain number of motor units on each $\mathrm{PCB}$

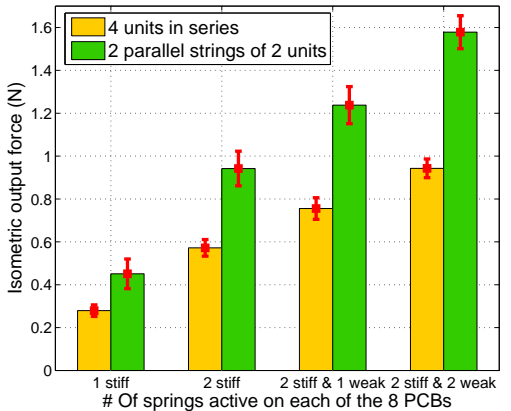

(b) Increasing the number of activated motor units on each PCB increases the output force.

Figure 13: Isometric output force experiments of $1 \times 4$ and $2 \times 2$ actuators.

$\mathrm{PCB}$ of the $1 \mathrm{x} 4$ actuator and the $2 \mathrm{x} 2$ actuator. In Fig. $13 \mathrm{~b}$ the output force is further increased by activating additional motor units on each PCB.

It is interesting to note that the results obtained are in accordance with the spring experiments shown in Fig.8a. Activating 1 weak motor unit on each $\mathrm{PCB}$, for example, results in an isometric output force of approximately $0.2 \mathrm{~N}$ and approximately $0.3 \mathrm{~N}$ for 1 strong motor unit.

\subsection{Open loop repeatability study}

Actuators consisting of a number of discrete motor units can benefit from their high repeatability without intensive control algorithms. The standard deviation in the experiments of Fig.13a and Fig.13b is, however, not totally negligible. The authors expect that repeatability will improve drastically with a more uniform manufacturing process, since currently the assembly is done by hand. A specific pattern for 1 weak motor unit on each PCB was activated 10 times. The same was done for an alternate activation pattern still consisting of weak motor unit on each PCB. The experiments were repeated for a strong motor unit as well. All experiments were done on the $1 \mathrm{x} 4$ actuator. The results are shown in Fig.14. The standard deviation of a specific activation pattern in Fig.14 is negligible which proves the high repeatability of a specific activation pattern. Therefore, it can be concluded that the standard deviations in Fig.13 
are due to the assembly by hand.

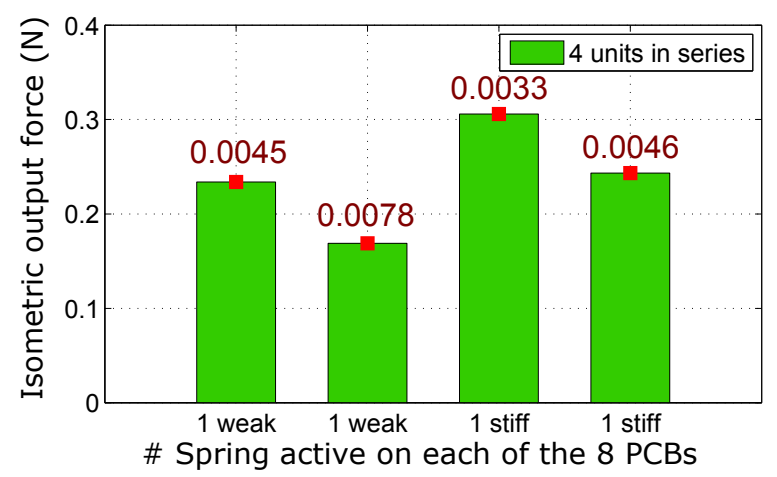

Figure 14: Redundant patterns do give a different output force due to manufacturing imprecision. However, this figure shows that activating a specific activation pattern multiple times is very repeatable.

\section{CONLUSION AND FUTURE WORK}

In a robot, the word "actuator" traditionally denotes a single servomotor or similar device. Human muscle, to the contrary, consists of several motor units, connected in series and parallel, which can be progressively recruited. In this paper, we present a novel design of a modular redundant actuation unit which can be combined in various combinations to form compliant actuators with characteristics derived from the intended function, much as muscle fibers are organized in human muscles. Inspired by the distinctive hierarchical structure of skeletal muscle, the compliant actuation unit presented in this paper is not only compliant, but also possesses a cellular structure. As such, multiple motor units cooperate to drive a single output. This paper presents the working principle and discusses the physical implementation in detail. The isometric experiments show the actuation characteristics and prove the high repeatability of the actuators composed of these actuation units. The platform can be used in future work to further explore the virtues of exploiting discretization and redundancy in muscle-like control. 
The actuation unit presented in this work consists of 12 motor units which can be activated independently. Each motor unit consists of a miniature solenoid to initiate contraction, and a compliant element. This work describes in detail the actuation unit implementation, which was particularly challenging due to the relatively small dimensions of the actuation unit. This includes close-packing of the solenoids in each actuation unit, as well as the design of a custom spring with 3 weak and 3 strong lobes. The design of a wire form spring and a leaf 540 spring are discussed in detail.

Two representative actuator configurations are evaluated experimentally, a chain of 4 units in series and a bundle of 2 parallel chains of 2 actuation units. Numerous other configurations are conceivable. The experiments corroborate the working principle and analytical models of the actuation units. Study of the repeatability of the output force as a function of the activation patterns showed high repeatability of the muscle-like actuator.

This actuator module has applications in humanoid robotics, rehabilitation robotics, anthropomorphic arms, etc. and gives roboticists the ability to produce "designer muscles" by combining these units in serial and parallel combinations to achieve specific properties. The authors, and their respective research groups, have particular interest in this actuator type for implementation in anthropomorphic arms with actuators build into the lower arm structure. Additionally, they are particularly useful in areas where high redundancy is needed, such as aerospace applications.

Future work includes refinement of the prototype, devising a more repeatable manufacturing process, further miniaturization, and investigation of discreteamplitude control strategies for force, displacement, and stiffness. In future designs of the actuation unit, special attention will go to improving the stress rate while remaining an equivalent strain rate. 


\section{Acknowlegdement}

This work was supported by ERC-291166-SOFTHANDS, ERC-337596-SPEAR and NSF-1427787-CNIC. Glenn Mathijssen is funded by PhD Fellowship of the Research Foundation - Flanders (FWO).

The authors would like to thank Phil Hudson, Marco Migliorini, Gianluca

Pane, Manuel Catalano, Nikos Tsagarakis and Stefano Cordasco for invaluable design suggestions and manufacturing support. The authors would like to thank Tom Van der Hoeven as well for his help with the experiments.

\section{References}

[1] G. A. Pratt, M. M. Williamson, Series elastic actuators, in: IEEE/RSJ International Conference on Intelligent Robots and Systems (IROS): Human Robot Interaction and Cooperative Robots, Vol. 1, 1995, pp. 399-406.

[2] R. Filippini, S. Sen, A. Bicchi, Toward soft robots you can depend on, IEEE Robotics \& Automation Magazine 10 (3) (2008) 31-41.

[3] A. Jafari, N. G. Tsagarakis, D. G. Caldwell, A novel intrinsically energy efficient actuator with adjustable stiffness (awas), IEEE/ASME Transactions on Mechatronics 18 (1) (2013) 355-365.

[4] B. Vanderborght, A. Albu-Schäffer, A. Bicchi, E. Burdet, D. Caldwell, R. Carloni, M. Catalano, O. Eiberger, W. Friedl, G. Gowrishankar, M. Garabini, M. Grebenstein, G. Grioloi, S. Haddadin, M. Laffranchi, H. Hoppner, A. Jafari, D. Lefeber, F. Petit, S. Stramigioli, N. Tsagarakis, M. Van Damme, R. Van Ham, L. V. Visser, S. Wolf, Variable Impedance Actuators: a Review, Robotics and Autonomous Systems 61 (12) (2014) $1601-11614$.

[5] A. Bicchi, G. Tonietti, M. Bavaro, M. Piccigallo, Variable stiffness actuators for fast and safe motion control, International Journal of Robotics Research (2005) 527-536. 
[6] B. Vanderborght, B. Verrelst, R. Van Ham, M. Van Damme, P. Beyl, D. Lefeber, Development of a compliance controller to reduce energy consumption for bipedal robots, Autonomous Robots 24 (4) (2008) 419-434.

[7] D. Villegas, M. Van Damme, B. Vanderborght, P. Beyl, D. Lefeber, Thirdgeneration pleated pneumatic artificial muscles for robotic applications: Development and comparison with mckibben muscle, Advanced Robotics 26 (11-12) (2012) 1205-1227.

[8] P. Brochu, Q. Pei, Advances in dielectric elastomers for actuators and artificial muscles, Macromolecular Rapid Communications 31 (1) (2010) 10-36.

[9] F. Carpi, R. Kornbluh, P. Sommer-Larsen, G. Alici, Electroactive polymer actuators as artificial muscles: are they ready for bioinspired applications?, Bioinspiration \& biomimetics 6 (4) (2011) 045006.

[10] E. Torres-Jara, K. Gilpin, J. Karges, R. Wood, D. Rus, Composable flexible small actuators built from thin shape memory alloy sheets, IEEE Robotics \& Automation Magazine 17 (4) (2010) 78-87.

[11] R. Enoka, Neuromechanics of Human Movement, 3rd Edition, Human Kinetics, Champaign, IL, 2002.

[12] E. Henneman, Relation between size of neurons and their susceptibility to discharge, Science 126 (3287) (1957) 1345-1347.

[13] K. Dittrich, Patent EADS Deutschland GmbH, Ottobrunn, DE: Cellular actuator device and methods of making and using the same (2006).

[14] D. MacNair, J. Ueda, Dynamic cellular actuator arrays and the expanded fingerprint method for dynamic modeling, Robotics and Autonomous Systems 62 (7) (2014) 1060 - 1072.

[15] D. Huston, B. Esser, G. Spencer, D. Burns, E. Kahn, Hierarchical actuator systems, Proceedings of SPIE 5762 (2005) 311-319. 
[16] G. Mathijssen, D. Lefeber, B. Vanderborght, Variable recruitment of parallel elastic elements: Series-parallel elastic actuators (spea) with dephased mutilated gears, IEEE Transactions on Mechatronics (in press) PP (2014) $1-9$.

[17] K. Cho, H. Asada, Architecture design of a multiaxis cellular actuator array using segmented binary control of shape memory alloy, IEEE Transactions on Robotics 22 (4) (2006) 831-843.

[18] J. Ueda, L. Odhner, H. H. Asada, Broadcast Feedback of Stochastic Cellular Actuators Inspired by Biological Muscle Control, The International Journal of Robotics Research 26 (11-12) (2007) 1251-1265.

[19] J. A. Schultz, J. Ueda, Nested piezoelectric cellular actuators for a biologically inspired camera positioning mechanism, IEEE Transactions on Robotics 29 (5) (2013) 1125-1138.

[20] Z. Butler, D. Rus, Distributed planning and control for modular robots with unit-compressible modules, The International Journal of Robotics Research 22 (9) (2003) 699-715.

[21] C. J. Paredis, H. Benjamin Brown, P. K. Khosla, A rapidly deployable manipulator system, Robotics and Autonomous Systems 21 (3) (1997) 289304.

[22] H. Oyobe, Y. Hori, Object conveyance system magic carpet? consisting of 64 linear actuators-object position feedback control with object position estimation, in: IEEE/ASME International Conference on Advanced Intelligent Mechatronics (AIM), Como, 2001, pp. 1307-1312.

[23] M. G. Catalano, G. Grioli, M. Garabini, F. Bonomo, M. Mancinit, N. Tsagarakis, A. Bicchi, Vsa-cubebot: A modular variable stiffness platform for multiple degrees of freedom robots, in: IEEE International Conference on Robotics and Automation (ICRA), 2011, pp. 5090-5095. 
${ }_{640}[24]$ H. Milner-brown, R. Stein, R. Yemm, The orderly recruitment of human motor units during voluntary isometric contractions, Journal of Physiology 230 (2) (1973) 359-370.

[25] J. Schultz, G. Mathijssen, B. Vanderborght, A. Bicchi, Toward motor-unitrecruitment actuators for soft robotics, in: RAS and EMBS International Conference on Biomedical Robotics and Biomechatronics (BioRob), 2014, pp. $887-892$.

[26] J. Ueda, T. W. Secord, H. H. Asada, Large effective-strain piezoelectric actuators using nested cellular architecture with exponential strain amplification mechanisms, IEEE/ASME Transactions on Mechatronics 15 (5) (2010) 770-782.

[27] R. Lieber, J. Friedn, Functional and clinical significance of skeletal muscle architecture, Muscle \& nerve 23 (2000) 1647-1666.

[28] J. M. Gere, B. J. Goodno, Mechanics of Materials, 8th Edition, Cengage Learning, Stamford, CT, 2013.

[29] L. Edström, E. Kugelberg, Histochemical composition, distribution of fibres and fatiguability of single motor units. Anterior tibial muscle of the rat., Journal of neurology, neurosurgery, and psychiatry 31 (5) (1968) 424-33.

[30] S. G. Laney, Symmetrical close packing of cylindrical objects, in: IEEE Systems and Information Engineering Design Symposium, 2014, p. (pagination not yet knwon).

[31] D. Neal, H. Asada, Bipolar piezoelectric buckling actuators, IEEE/ASME Transactions on Mechatronics 19 (1) (2014) 9-19.

[32] M. Goldberg, Packing of 14, 16, 17 and 20 circles in a circle, Mathematics Magazine 44 (3) (1971) 134-139.

665 [33] G. Reis, Dense packing of equal circles within a circle, Mathematics Magazine 48 (1) (1975) 33-37. 
[34] R. C. Juvinall, K. M. Marshek, Fundamentals of machine component design, Vol. 83, John Wiley \& Sons New York, 2006.

[35] J. Madden, N. Vandesteeg, P. Anquetil, P. Madden, a. Takshi, R. Pytel, S. Lafontaine, P. Wieringa, I. Hunter, Artificial Muscle Technology: Physical Principles and Naval Prospects, IEEE Journal of Oceanic Engineering 29 (3) (2004) 706-728. 


\section{Glenn Mathijssen}

Glenn Mathijssen received the degree in the study of Electro-Mechanical Engineering at the Vrije Universiteit Brussel (VUB), Belgium in 2012. Currently he is PhD candidate in a Joint PhD between the Robotics and Multibody Mechanics research group (R\&MM) at VUB, and Centro E. Piaggio at the University of Pisa, Italy. He is funded by PhD Fellowship of the Research Foundation - Flanders (FWO), under the supervision of Prof. Dr. Ir. Bram Vanderborght, and co-supervision of Prof. Dr. Ir. Dirk Lefeber and Prof. Antonio Bicchi. His research interests include compliant actuation, novel actuators and energy efficient actuation.

\section{Joshua Schultz}

Joshua Schultz received the B.S. degree from Tufts University, Medford, MA, USA, in 2002, the M.S. degree from Vanderbilt University, Nashville, TN, USA, in 2004, and the Ph.D. degree from the Woodruff School of Mechanical Engineering, Georgia Institute of Technology, Atlanta, GA, USA, in 2012, all in mechanical engineering.

From 2004 to 2008, he was a member of the Power Systems and Motion Control Group, Printing Services and Solutions Division, Lexmark International, Lexington, KY, USA. From 2012 to 2013, he was a Postdoctoral Fellow with the Department of Advanced Robotics, Istituto Italiano di Tecnologia, Genoa, Italy. Since 2013, he has been with the Department of Mechanical Engineering at the University of Tulsa, Tulsa, OK, USA as an Assistant Professor. His current research interests include system dynamics of biological systems, nonlinear control, soft robotics, underactuted grasping with compliant hands, and nontraditional distributed sensing and actuation technologies.

Dr. Schultz is a recipient of the 2011 Achievement Rewards for College Scientists Foundation Scholarship.

\section{Bram Vanderborght}

Prof. dr. ir. Bram Vanderborght received his PhD in 2007. The focus of his research was the use of adaptable compliance of pneumatic artificial muscles in the dynamically balanced biped Lucy. In 2006 he performed research on the humanoids robot HRP-2 in AIST, Tsukuba (Japan). From October 2007April 2010 he worked as post-doc researcher at IIT (Italy). Since October 2009, he is appointed as professor at the VUB. He is member of the Young Academy of the Royal Flemish Academy of Belgium for Science and the Arts. In 2013 he received an ERC Starting Grant on new generation compliant actuators. His research interests include cognitive and physical human robot interaction with core technology using variable impedance actuators.

\section{Antonio Bicchi}

Antonio Bicchi is Professor of Robotics at the University of Pisa, and Senior Scientist at the Italian Institute of Technology in Genoa. He graduated from the University of Bologna in 1988 and was a postdoc scholar at M.I.T. Artificial Intelligence lab in 1988-1990. He teaches Control Systems and Robotics in the Department of Information Engineering (DII) of the University of Pisa, leads the Robotics group at the Research Center "E. Piaggio" of the University of Pisa since 1990, and served 
as Director from 2003 to 2012. He is an Adjunct Professor at the School of Biological and Health Systems Engineering of Arizona State University since 2013.

His main research interests are in Robotics, Haptics, and Control Systems in general. He has published more than 300 papers on international journals, books, and refereed conferences. He served as the President of the Italian Association or Researchers in Automatic Control in 2012-2013, as Editor in Chief of the Conference Editorial Board for the IEEE Robotics and Automation Society (RAS), as Vice President and as Distinguished Lecturer of IEEE RAS. He is Editor-in-Chief for the book series "Springer Briefs on Control, Automation and Robotics," and is in the editorial board of several scientific journals, including the top-ranked Int.I J. Robotics Research, the IEEE Trans. on Robotics and Automation, IEEE Trans. Automation Science and Engineering, and IEEE RAS Magazine. He has organized and co-chaired the first WorldHaptics Conference (2005), and Hybrid Systems: Computation and Control (2007). He is the recipient of several awards and honors. In 2012, he was awarded with an individual Advanced Grant from the European Research Council for his research on human and robot hands. Antonio Bicchi is a Fellow of IEEE since 2005. 


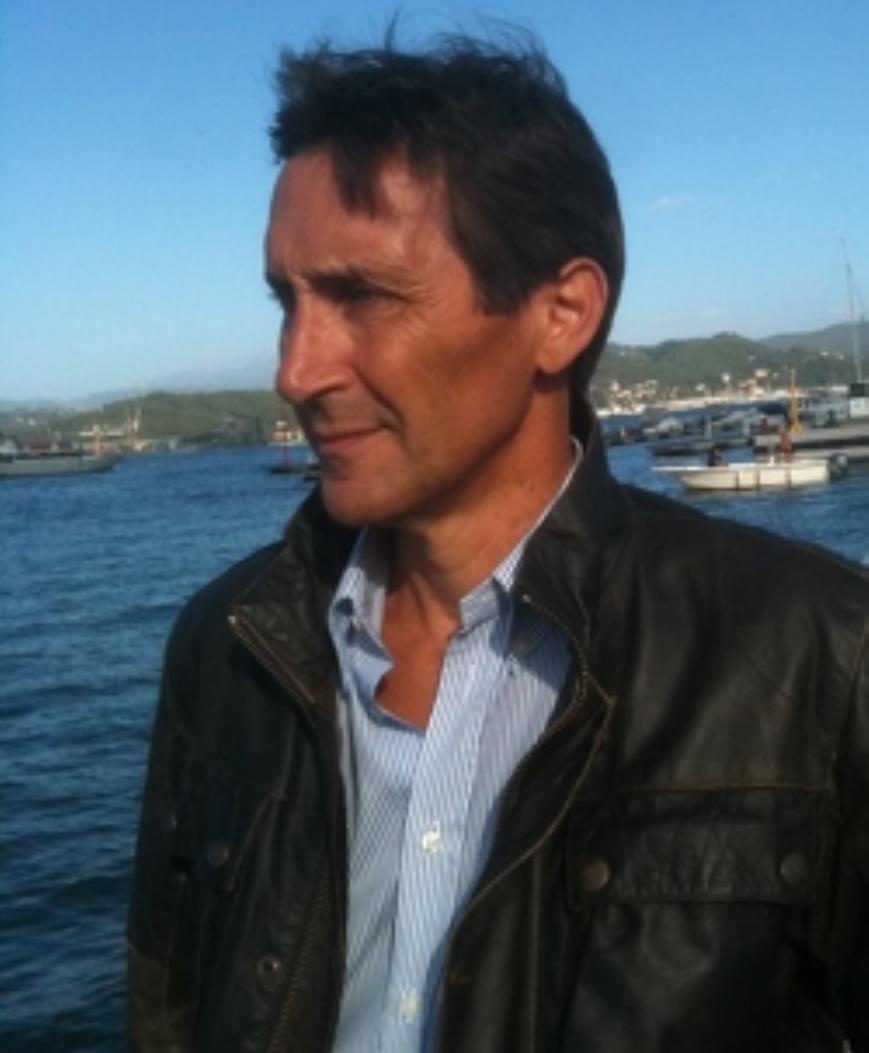




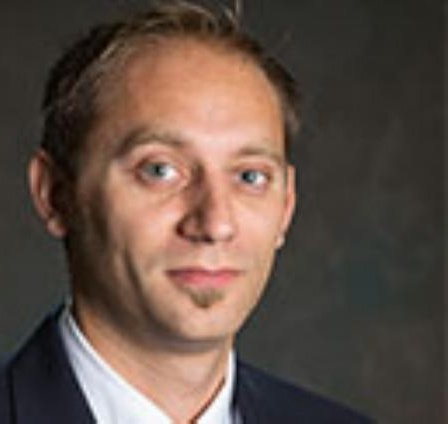





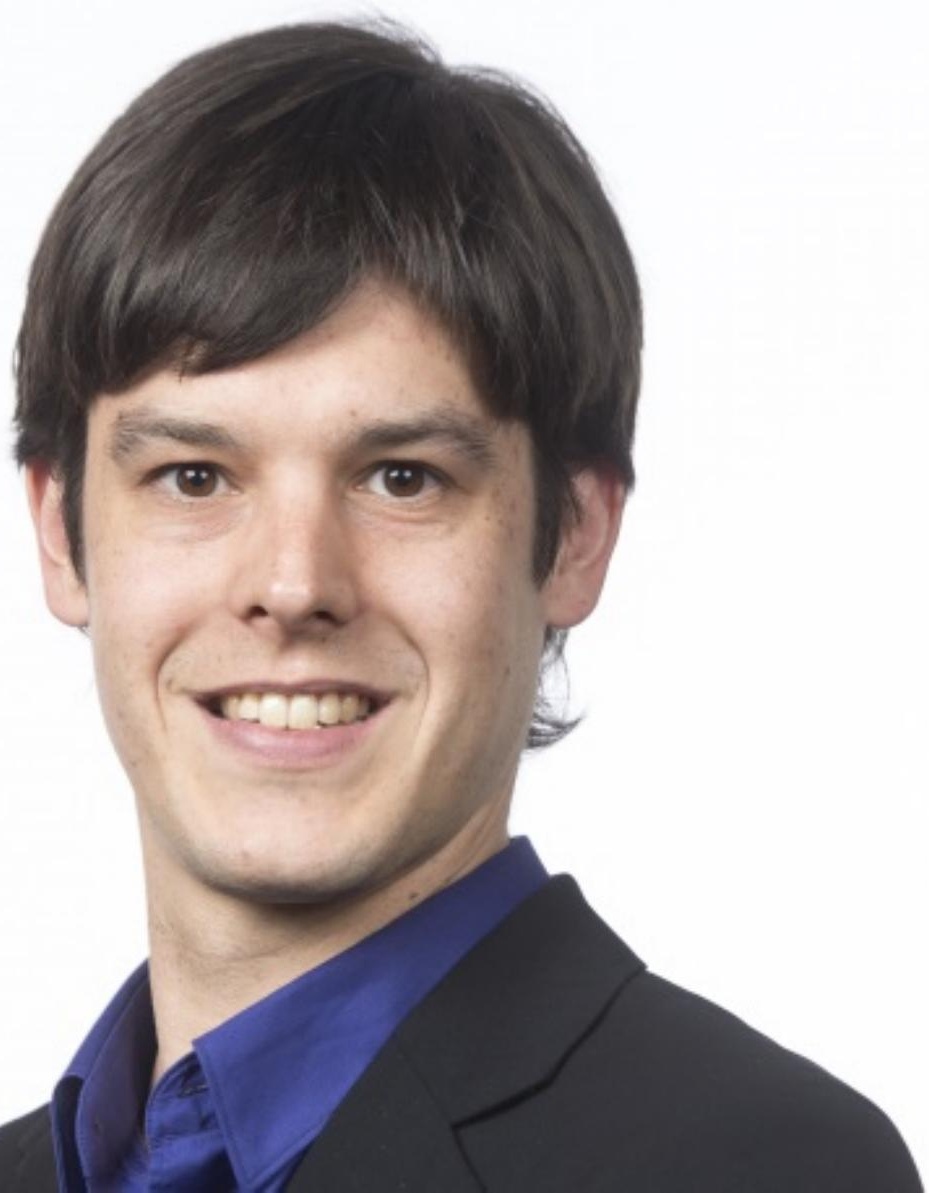

